\title{
Administrador y las megatendencias de la nueva estructura demográfica, familiar y educación personalizada
}

\author{
Administrator and the megatrends of the new demographic, family structure and personalized \\ education \\ José Luis Alvarado-Resendiz ${ }^{a}$, Mónica García-Munguía ${ }^{b}$, Liliana Yadira Castellano-López $^{c}$, \\ Edgar Sarabia-Lugo ${ }^{d}$
}

\begin{abstract}
:
At present, globalization generates a complexity of the environment, in the speed and magnitude of the changes that occur specifically in global markets, forcing the administrator to perform a constant analysis of the new global and regional conditions. The importance of the identification and analysis of mega-managements by the administrator lies not only in the prevention of future events established by the markets. This research examines the new challenges of the administrator, with the megatrends of demographic, family st ructure and personalized education, showing the importance and impact in the scope of their abilities. The main purpose is to assimilate the premises of the megatrends of a society and the impact on the business activity that the administrator faces, in order to know and update the broader and more accurate perception, without forgetting that they offer information to detect the exploitation of opportunities established by the global market.
\end{abstract}

Keywords:

Manager, megatrends, future

\section{Resumen:}

En la actualidad la globalización genera una complejidad del entorno, en la velocidad y magnitud de los cambios que se presentan específicamente en los mercados globales, obligando al administrador a realizar un análisis constante de las nuevas condiciones globales y regionales. La importancia de la identificación y análisis de las megatendecias por parte del administrador, radica no solo en la prevención de los acontecimientos futuros que establecen los mercados. Esta investigación examina los nuevos desafíos del administrador, con las megatendencias de estructura demográfica, familiar y educación personalizada, mostrando la importancia e impacto en el ámbito de sus habilidades. El fin principal es asimilar las premisas de las megatendencias de una sociedad y el impacto en la actividad empresarial a la que se enfrenta el administrador, con el propósito de conocer y actualizarse de la percepción más amplia y precisa, sin olvidar que ofrecen información para detectar el aprovechamiento de oportunidades que establece el mercado global..

\section{Palabras Clave:}

Administrador, megatendencias, futuro

\footnotetext{
Autor de Correspondencia, Universidad Autónoma del Estado de Hidalgo, Escuela Superior de Tlahuelilpan., email: jose_alvarado4225@uaeh.edu.mx.

b Universidad Autónoma del Estado de Hidalgo, Escuela Superior de Tlahuelilpan, https://orcid.org/0000-0002-0507-3933, email: monicagm@uaeh.edu.mx

c Instituto Tecnológico Superior del Occidente del Estado de Hidalgo. email: lcastellanos@itsoeh.edu.mx

d Instituto Tecnológico Superior del Occidente del Estado de Hidalgo, email: esarabia@itsoeh.edu.mx
} 


\section{Introducción}

La globalización y el desarrollo económico mundial, plantean al entorno empresarial un conjunto de retos que hacen que las empresas tengan que comprender las alternativas de actuación más aptas, por ende, entre otros factores la organización tendrá que acostumbrarse a desenvolverse en un ambiente de mayor confusión estratégica y con una creciente presencia de empresas en los mercados.

Los cambios que establecen los mercados se presenta en todas las industrias y zonas geográficas, impulsados por la convergencia de lo social con lo móvil, la nube, los grandes volúmenes de datos y la demanda cada vez con mayor facilidad de acceso a la información en cualquier momento y en cualquier lugar, en donde la educación y nueva estructura demográfica está transformando constantemente la participación en el mercado global a todas las áreas de las empresas. Existen grandes oportunidades para que las empresas, se enfrenten a un primer estudio a las megatendencias de la educación y la estructura demográfica, aprovechando los dispositivos conectados, habilitados por el internet de las cosas, para capturar grandes cantidades de información, facilitando el ingreso a nuevos mercados, y la transformación de los productos existentes e introducir nuevos modelos de negocios y de entrega al mercado competitivo.

Las megatendencia se pueden describir como elementos que permiten aproximarse al conocimiento sobre el comportamiento en el futuro de los factores que caracterizan a las personas y empresas, sin olvidar la participación del administrador, quien desarrolla la gestión organizacional, se ha consensado que se pueden definir como un mecanismo que permite tener en cuenta el mañana, y poder cambiar el presente con el fin de obtener resultados efectivos que son procedentes de hechos actuales que se vislumbran como el óptimo deseable de la empresa, es decir, que debe primero ajustarse hoy el conocimiento para generar el éxito en un determinado periodo de tiempo. Esta investigación analiza los nuevos desafíos del administrador, con las megatendencias de estructura demográfica, familiar y educación personalizada, mostrando la importancia e impacto en el ámbito de sus habilidades.

Los administradores se deben preparase para asumir la constante y creciente necesidad de información y los cambios en su propia preparación, para estar en la progreso de las decisiones de competencia económica y productiva, porque la organización según Valdés (2005), se interrelaciona con otro macrosistema llamado entorno, en donde se mueve la empresa y se hallan diferentes elementos que la afecten, y considerarse parte de lo mismo.

\section{Desarrollo}

El mundo globalizado advierte todos los días el conocimiento e innovación como eje central de la generación de riqueza, aconteciendo nuevas formas de organización social y estructuras organizacionales que ofrezcan oportunidad y rapidez en la entrega de bienes y servicios; además la información, la tecnología y el capital humano se posicionan como cimientos fundamentales de procesos operativos, de las actividades laborales y trabajo directivo.

El mundo globalizado de acuerdo con González O., González O., Ríos y León (2013), están en constante revuelta, está haciendo que las instituciones sociales y en especial las de educación superior, se encuentren modernizándose desde las perspectivas académicas, para mantenerse en la evolución de las exigencias y desafíos provenientes de la sociedad del conocimiento. Diariamente, la era del conocimiento se incrementa invadiendo cualquier rincón del planeta, motivo que lleva a efectuar un primer análisis en la investigación sobre la megatendencia de la educación personalizada, vitalicia y universal; así mismo de la Nueva estructura demográfica y familiar, generadas por la globalización

La globalización no se puede detener, pero si puede advertir con profunda disposición al conocimiento, innovación y tecnología, principalmente por los administradores de las organizaciones. En la confusión de la administración actual el administrador es la persona que representa al empresario frente a los trabajadores. Por tanto, si el administrador suple en sus funciones al empresario o dueño de la organización, para que su trabajo sea eficaz requiere de un conocimiento vasto de la visión y las necesidades empresariales. La diferencia principal para distinguir entre el administrador y el empresario es la posesión del capital, pero en cuanto a conocimientos, el administrador demanda tener los mismos o más conocimientos que el empresario, para colaborar o sustituirle en la dirección de las organizaciones.

El desafío de la administración de empresas en la actualidad, presenta varias líneas: satisfacer a un cliente que cuenta con diversas opciones en el mundo, ser muy eficiente en el manejo de los recursos, crear empleos y satisfacción para sus colaboradores en la mejora de su calidad de vida, competir con otras organizaciones de mayor capital para anhelar el liderazgo del mercado, y finalmente, formar personas que forjen valor todos los días a la organización y a la sociedad. Entendiendo que la administración según Münch y García (2008), es el proceso cuyo objeto es la coordinación eficaz y eficiente 
de los recursos de una organización para lograr sus objetivos planteados con la máxima productividad, eficiencia y calidad; por lo tanto, se debe mantener en un proceso de permanente innovación y dinamismo, acorde con los progresos científicos y tecnológicos generados por los rápidos cambios que advierte el entorno productivo, económico, social y cultural del mundo.

La nueva economía del conocimiento, obliga a que el administrador deba ser el primer interesado en su utilización, buscando la forma de conocerse, formarse y venderse, ante ese nuevo reto. Para ello el administrador se debe actualizar en todo momento, movilizándose a la rapidez de los acontecimientos del entorno global, y las circunstancias por las que atraviesa el mundo de la administración, procurando ser para cualquier organización altamente productivo. Es importante destacar que es viable para el administrador según Mojica (2005), que construir el futuro no debe estar cimentado únicamente en el análisis interno de una organización o territorio; puede realizar una mejor tarea si conoce las reglas con las que está jugando el mercado global, y no poder hacer caso omiso a las líneas de fuerza y tendencias mundiales que acompañarán probablemente, durante muchos años.

En la actualidad se tiene educación por competencias, en donde se desarrollan conocimientos, habilidades, actitudes y valores para la vida y el trabajo; insistiendo en que el profesional de la administración deberá tener apertura mental, flexibilidad y creatividad para generar empleos y oportunidades, que establecen las exigencias de los mercados. La búsqueda de la calidad en la enseñanza hace que cada día, los centros educativos se sientan involucrados con la necesidad de cambio en todo su hacer, fundamentalmente en lo que se refiere al liderazgo desarrollado por sus docentes de los futuros administradores, en su desempeño laboral.

En el ámbito educativo, según el Centro Nacional de Planeamiento Estratégico (CEPLAN, 2014), como muchas actividades actuales, se ha visto fuertemente influido por la incorporación de las Tecnologías de Información y comunicaciones (TIC), de manera tal que puede afirmarse que es uno de los principales detonadores que están generando cambios en el acceso a la información. Sin embargo, existe un entorno de demandas económicas y sociales que están impactando fuertemente la evolución y posible situación futura de la Educación. Los detonadores e impulsores: Tecnologías de Información y comunicaciones (TIC), es decir, transmisión de la información mediante medios de comunicación, cambios en la demanda del mercado laboral, y cambio en la pirámide poblacional.
Una habilidad del administrador es tener una percepción más amplia y precisa de por qué los problemas, y de acuerdo a Atwater, Kannan y Stephens (2008), las organizaciones son ahora reconocidas como parte de un sistema más grande con propósito, es decir, los diversos tipos de sociedad mercantil, con muchos subsistemas o áreas funcionales y las partes, como es el caso de los colaboradores, en la búsqueda de cumplir sus propios objetivos individuales. El administrador debe considerar de manera importante el papel de la educación, por estar llegando más allá del desarrollo de los conocimientos y las competencias cognoscitivas para la construcción de valores, competencias y actitudes entre los alumnos, motivo que lleva a la educación a ser el impulsor del proceso de transformación, que implican a el alumno en el análisis crítico sobre las complicadas cuestiones de alcance mundial, y en el desarrollo de competencias como la comunicación, cooperación y resolución de conflictos, entre otras, para resolver los problemas de cualquier organización.

En las exigencias del mundo globalizado, la educación se debe poner mayor vigor en preparar a las personas desde una edad temprana y a lo largo de toda su vida, con los conocimientos, competencias, actitudes y comportamientos que se necesitan para ser ciudadanos informados, comprometidos y solidarios, en la solución de problemas o al enfrentar la proyección del mundo de la educación según Centro Nacional de Planeamiento Estratégico (CEPLAN, 2014):

- La creatividad y la innovación son esenciales para su desarrollo e inherentes a la conciencia humana

- Implementar acciones en las que la distancia deje de ser un condicionante; es así que la utilización de sistemas multimedia

- La conceptualización de la educación se vea como un proceso que se extiende a lo largo de la vida y que el hecho de que la educación a distancia pueda considerarse como una alternativa real a la educación presencial.

- $\quad$ Se hace necesario enfatizar que los beneficios de la sociedad de la información para los países en vías de desarrollo

La educación es una herramienta que permite acceder a la participación internacional y promoviendo la transformación social en una forma innovadora para lograr un mundo justo y pacífico, tolerante, inclusivo, seguro y sostenible, para las nuevas generaciones. La educación, mejora la base documental, el fortalecimiento de la colaboración y la construcción de coaliciones, en donde 
tienen que trabajar en el respeto por los demás y por el mundo en que vivimos, ayudando a la gente a crear sociedades justas, comprensivas y pacíficas. Por eso, es importante destacar los elementos clave que señala CEPLAN (2014), de la megatendencia de la educación: Enseñanza, Aprendizaje, Seguimiento, Desarrollo del Ser Humano, Alumno, Profesor, Institución, Sociedad, Currículo, Evidencias de evaluación del aprendizaje, Oferta formativa, Educación básica, Proceso educativo, Objeto de enseñanza, Objetivos educativos, Incorporación de las TICs, Utilaje educativo y Evaluación del aprendizaje.

Al analizar el administrador la megatendencia de la educación personalizada, vitalicia y universal prospectiva, le permitirá a ayudar e mejorar la toma de decisiones al estar informado en el presente, proponiendo una variedad relevante de trayectorias de futuro, un futuro que está abierto, incierto, indeterminado, y que no se puede conocer anticipadamente con certeza.

Otra megatendencia que el administrador debe identificar, citando a CEPLAN (2014), el continuo incremento de la población, el cambio de su estructura, el proceso de envejecimiento y la creciente urbanización. $Y$ si los administradores conocen, la reducción de las tasas globales de fecundidad, la menor presencia de población joven en las estructuras de población, el dinamismo de los procesos migratorios globales y el acelerado proceso de urbanización de los países en desarrollo, entre otros, les permitirá enfrentar los constantes cambios que tiene el mercado global, y de igual forma podrían aprovechar las oportunidades para el desarrollo que estén inmersas estos fenómenos y, al mismo tiempo, prevenir futuras tensiones vinculadas con la distribución adecuada de recursos, la demanda de mano de obra, la generación de servicios y oportunidades para la población y otras cuestiones que indefectiblemente redundan en su bienestar.

A los administradores prepararse para los cambios del mañana de acuerdo a Ferris (2011), significa no solamente establecer mecanismos de envío de socorros y estrategias de protección más enérgicos, sino también analizar los ambientes políticos y económicos que determinará la naturaleza de las barreras del futuro. Es importante para el administrador analizar megatendencias que probablemente van a delinear el contexto en el que se desarrollen las intrusiones humanitarias en el transcurso de los próximos veinticinco años y se intenta deducir sus secuelas para la acción humanitaria.

La estructura demográfica y familiar ha cambiado, parafraseando a CEPLAN (2014), en esta época de globalización e intensa migración, los detonadores de la nueva estructura familiar, y de la pirámide poblacional se haya modificado a razón de:

- Los incentivos de gobierno para la educación de las mujeres contribuyen a que haya una mayor inserción de las mismas al mercado laboral provocando que tengan menos hijos.

- La mejora de las Tecnologías de Información y comunicación, que ahora es posible encontrar casi en cualquier lugar ha facilitado que haya nuevas formas de trabajo, que minimizan los traslados y ofrecen la posibilidad de trabajar desde casa.

- Los medios de Información: Medios Impresos, Radio, Televisión, Internet difunden y permiten conocer más temas sobre salud, anticonceptivos y bienestar en general lo que provoca la planificación familiar y el mejor cuidado de la salud, esto ha llevado a que aumente la esperanza de vida y a que personas de 65 años estén sanas y con posibilidades de laborar lo que lleva a pensar en nuevas edades para la jubilación.

Desde esta perspectiva demográfica, surgirán ciudades viejas y nuevas, que enfrentarán retos de mercado global. Las poblaciones jóvenes podrán ayudar a crear fuerzas laborales productivas, aunque también pueden provocar problemas en países con altos niveles de subempleo y otros males sociales, por el incremento poblacional. Desafortunadamente las poblaciones que están envejeciendo se retiran de la fuerza laboral sin que haya personas con las capacidades y experiencias apropiadas para sustituirlas, debilitando el crecimiento y afectando los recursos públicos para su abastecimiento.

Las proyecciones de población se deberán convertir en un instrumento indispensable para el administrador, en la formulación e instrumentación de acciones y programas, permitiendo anticiparse a las necesidades sociales y situaciones que podrían convertirse en inconvenientes, así como canalizar recursos de diverso tipo para su atención. Analizar la transición demográfica es plantear los desiguales ritmos y evolución de las variables del cambio demográfico, citando a CEPLAN (2014), es conveniente identificar los elementos que propician estos cambios:

- La migración del campo a las ciudades ha aumentado y la tendencia a que siga creciendo es considerable.

- La educación de la mujer ha aumentado, cada día encontramos mayor número de mujeres en la educación media y media superior provocando que el desempeño y 
el número de mujeres con mayor educación y mejores habilidades aumenten considerablemente.

- Nuevas Formas de Trabajo donde se espera que los empleados no necesiten asistir a un lugar para trabajar, lo cual logrará que haya una pérdida de sentido del tradicional día laboral.

- $\quad$ Pensar en nuevas edades para el retiro, nuevas formas de fondos de pensiones, y un incremento considerable en la demanda de servicios como spas de longevidad, cirugías plásticas y de cuidado de la salud.

- Los medios de Información tendrán mayor cobertura en temas relacionados con la salud y la alimentación, lo que provoca que las personas se preocupen cada vez más y a más temprana edad sobre estos temas.

La política de población, de acuerdo con Hernández, López, y Velarde (2013), tiene como uno de sus soportes técnicos los insumos que le ofrece la prospectiva demográfica, con éstos es posible la formulación de estrategias y líneas de acción de los administradores sobre mortalidad, fecundidad y salud reproductiva, migración interna e internacional, envejecimiento, bono demográfico, entre otros, para su toma de decisiones empresariales.

Si bien la urbanización ofrece oportunidades económicas, también presenta considerables riesgos relacionados con los recursos. El cambio demográfico desarrolla oportunidades, desafíos extraordinarios y el panorama para los administradores para estar preparadas en un mundo que es cada día más competitivo y con caminos tecnológicos globales e imponentes. En la nueva era de la globalización y las megatendencias de la nueva estructura demográfica, familiar y educación personalizada, es necesario para el administrador salir de la tecnificación y construir negocios basado en la información social centrándose en representar valor para los usuarios con una reingeniería en la forma de participar en los mercados competitivos.

\section{Conclusiones}

Las organizaciones y los administradores del presente están en un terreno de negocios sensible que enfrenta cambios constantemente, llevándolos a un alto nivel de riesgo en dos aspectos fundamentales: la sobrevivencia de los negocios y la incertidumbre en la inversión.

La subsistencia y sobrevivencia en un entorno de competencia y globalizador determina un desarrollo tanto estable como creciente para todos las organizaciones, parte de esta temática implica un riesgo constante en donde la toma de decisiones de los administradores que debe proteger los intereses de los negocios, haciendo necesario que concuerden con las tendencias mundiales y regionales para trabajar dentro del margen de los mercados y economías del futuro.

La solución a los desafíos planteados son verdaderos retos de futuro para la humanidad, deben ser abordados de manera conjunta por los administradores, porque la empresa juega un papel importante en la solución de dichos conflictos, por ser un generador de crecimiento económico. La posibilidad que tiene una empresa de generar inclusión social, cambios culturales e incidir en la conducta de la sociedad, debe ser aprovechada por el administrador buscando un mundo que logre el beneficio económico, y distribuya de manera más equitativa, permitiendo el bienestar de la humanidad, y de inicio considerar las megatendencias de la nueva estructura demográfica, familiar y educación personalizada, por parte del administrador para enfrentar los retos en materia de educación y cambios demográficos, con el único propósito de establecer estrategias que permita afrontar los cambios constantes que plantea la globalización

El propósito de la prospectiva es abrir, a ojos de los administradores, el abanico de futuras posibilidades, imaginar cambios en las estructuras, detectar disponibilidades presentes de inicio en materia de educación, reflexionando según CEPLAN (2014), en educación a distancia tradicional y educación presencial, educación no-virtual y la virtual, como medio para articularse en un nuevo ambiente de intensa interacción entre los actores que intervienen en el proceso de enseñanza-aprendizaje, y los procesos de las instituciones de educación superior, con el único propósito de formar a los administradores que dirigirán a las organizaciones del futuro.

La eliminación de todo tipo de discriminación, la equiparación de las oportunidades para las personas menos favorecidas, las buenas prácticas de gobierno y la transparencia en la acción de las organizaciones, son en gran parte responsabilidad de las empresas, de sus administradores, y sus integrantes en general, quienes no pueden seguir impidiendo la responsabilidad en la construcción del futuro social, político y económico, considerando la megatendencia de la estructura demográfica y familiar, porque su expansión ha permitido el aumento en los ingresos reales de amplios sectores de la población, difusión y aplicación de los últimos avances médicos, el mejoramiento del estado nutricional de la población y su mayor acceso a la atención de salud y a la educación. 


\section{Referencias}

[1] Atwater B. J., Kannan V. R. \& Stephens A. A. (2008). Cultivating Systemic Thinking in the Next Generation of Business Leaders. Academy of Management Learning \& Education. Vol. 7. Utah State University

[2] Centro Nacional de Planeamiento Estratégico (2014). Megatendencias: un análisis del estado global. Primera edición. Recuperado de: https://www.ceplan.gob.pe/wp-

content/uploads/2016/08/Megatendencias-Un-an\%C3\%A1lisis-delestado-global-Ceplan.pdf

[3] Ferris E. (2011). Las megatendencias y el futuro de la acción humanitaria. International Review of de Red Cross. N. ${ }^{\circ} 884$ de la versión Recuperado de: file:///C:/Users/Jos\%C3\%A8\%20Luis/Downloads/irrc-884-ferris.pdf

[4] González O., González O., Ríos G. y León J. (2013). Características del liderazgo transformacional presentes en un grupo de docentes universitario Telos, vol. 15, núm. 3, septiembre-diciembre, 2013, pp. 355-371 Universidad Privada Dr. Rafael Belloso Chacín Maracaibo, Venezuela. Recuperado http://www.redalyc.org/pdf/993/99328424005.pdf

[5] Hernández López M. F., López Vega R., y Velarde Villalobos S. I. (2013). La situación demográfica en México. Panorama desde las proyecciones de población. La situación demográfica de México. Recuperado de: http://www.conapo.gob.mx/work/models/CONAPO/Resource/1720/1/i mages/1_La_Situacion_Demografica_En_Mexico.pdf

[6] Mojica F. J. (2005). La construcción del futuro. Concepto y modelo de prospectiva estratégica, territorial y tecnológica. Editorial U. Externado de Colombia. ISBN: 9789586169295. Categoría Investigación y Ciencia.

[7] Münch G. L. y García J. (2008). Fundamentos de administración. Editorial Trillas. México D.F.

[8] Valdés Hernández L. A. (2005). Planeación Estratégica con Enfoque Sistémico. Editorial FOCOA. México D.F. 